Sitong Chen and Xianhua Tang*

\title{
Berestycki-Lions conditions on ground state solutions for a Nonlinear Schrödinger equation with variable potentials
}

https://doi.org/10.1515/anona-2020-0011

Received December 28, 2018; accepted January 7, 2019.

Abstract: This paper is dedicated to studying the nonlinear Schrödinger equations of the form

$$
\left\{\begin{array}{l}
-\triangle u+V(x) u=f(u), \quad x \in \mathbb{R}^{N} ; \\
u \in H^{1}\left(\mathbb{R}^{N}\right),
\end{array}\right.
$$

where $V \in \mathcal{C}^{1}\left(\mathbb{R}^{N},[0, \infty)\right)$ satisfies some weak assumptions, and $f \in \mathcal{C}(\mathbb{R}, \mathbb{R})$ satisfies the general BerestyckiLions assumptions. By introducing some new tricks, we prove that the above problem admits a ground state solution of Pohožaev type and a least energy solution. These results generalize and improve some ones in [L. Jeanjean, K. Tanka, Indiana Univ. Math. J. 54 (2005), 443-464], [L. Jeanjean, K. Tanka, Proc. Amer. Math. Soc. 131 (2003) 2399-2408], [H. Berestycki, P.L. Lions, Arch. Rational Mech. Anal. 82 (1983) 313-345] and some other related literature. In particular, our assumptions are "almost" necessary when $V(x) \equiv V_{\infty}>0$, moreover, our approach could be useful for the study of other problems where radial symmetry of bounded sequence either fails or is not readily available, or where the ground state solutions of the problem at infinity are not sign definite.

Keywords: Schrödinger equation; Ground state solution of Pohožaev type; The least energy solution; Berestycki-Lions conditions

MSC: 35J20, 35J65

\section{Introduction}

In this paper, we consider the nonlinear Schrödinger equations of the form:

$$
\left\{\begin{array}{l}
-\triangle u+V(x) u=f(u), \quad x \in \mathbb{R}^{N} ; \\
u \in H^{1}\left(\mathbb{R}^{N}\right),
\end{array}\right.
$$

where $N \geq 3, V: \mathbb{R}^{N} \rightarrow \mathbb{R}$ and $f: \mathbb{R} \rightarrow \mathbb{R}$ satisfy the following basic assumptions:

(V1) $\quad V \in \mathcal{C}\left(\mathbb{R}^{N},[0, \infty)\right)$;

(V2) $\quad V(x) \leq V_{\infty}:=\lim _{|y| \rightarrow \infty} V(y)$ for all $x \in \mathbb{R}^{N}$;

(F1) $\quad f \in \mathcal{C}(\mathbb{R}, \mathbb{R})$ and there exists a constant $\mathcal{C}_{0}>0$ such that

$$
|f(t)| \leq \mathcal{C}_{0}\left(1+|t|^{2^{*}-1}\right), \quad \forall t \in \mathbb{R} ;
$$

$$
f(t)=o(t) \text { as } t \rightarrow 0 \text { and }|f(t)|=o\left(|t|^{(N+2) /(N-2)}\right) \text { as }|t| \rightarrow+\infty \text {. }
$$

Sitong Chen, School of Mathematics and Statistics, Central South University, Changsha, Hunan 410083, P.R.China, E-mail: mathsitongchen@163.com

*Corresponding Author: Xianhua Tang, School of Mathematics and Statistics, Central South University, Changsha, Hunan 410083, P.R.China, E-mail: tangxh@mail.csu.edu.cn 
Clearly, under (V1), (V2), (F1) and (F2), the weak solutions of (1.1) correspond to the critical points of the energy functional defined in $H^{1}\left(\mathbb{R}^{N}\right)$ by

$$
I(u)=\frac{1}{2} \int_{\mathbb{R}^{N}}\left[|\nabla u|^{2}+V(x) u^{2}\right] \mathrm{d} x-\int_{\mathbb{R}^{N}} F(u) \mathrm{d} x,
$$

where $F(t):=\int_{0}^{t} f(s) \mathrm{d} s$.

If the potential $V(x) \equiv V_{\infty}$, then (1.1) reduces to the following autonomous form:

$$
\left\{\begin{array}{l}
-\triangle u+V_{\infty} u=f(u), \quad x \in \mathbb{R}^{N} ; \\
u \in H^{1}\left(\mathbb{R}^{N}\right),
\end{array}\right.
$$

its energy functional is as follows:

$$
I^{\infty}(u)=\frac{1}{2} \int_{\mathbb{R}^{N}}\left(|\nabla u|^{2}+V_{\infty} u^{2}\right) \mathrm{d} x-\int_{\mathbb{R}^{N}} F(u) \mathrm{d} x .
$$

It is well known that every solution $u(x)$ of (1.3) satisfies the following Pohožaev type identity [9]:

$$
\mathcal{P}^{\infty}(u):=\frac{N-2}{2}\|\nabla u\|_{2}^{2}+\frac{N V_{\infty}}{2}\|u\|_{2}^{2}-N \int_{\mathbb{R}^{N}} F(u) \mathrm{d} x=0 .
$$

Let

$$
\mathcal{M}^{\infty}:=\left\{u \in H^{1}\left(\mathbb{R}^{N}\right) \backslash\{0\}: \mathcal{P}^{\infty}(u)=0\right\} .
$$

Berestycki-Lions [1] proved that (1.3) has a radially symmetric positive solution provided $f$ satisfies (F1), (F2) and the following two assumptions:

(F0) $\quad f$ is odd;

(F3) there exists $s_{0}>0$ such that $F\left(s_{0}\right)>\frac{1}{2} V_{\infty} s_{0}^{2}$.

To prove the above result, Berestycki-Lions [1] considered the following constrained minimization problem

$$
\min \left\{\|\nabla u\|_{2}^{2}: u \in \mathcal{S}\right\},
$$

where

$$
\mathcal{S}=\left\{u \in H^{1}\left(\mathbb{R}^{N}\right): \int_{\mathbb{R}^{N}}\left[F(u)-\frac{1}{2} V_{\infty} u^{2}\right] \mathrm{d} x=1\right\} ;
$$

they first showed that by the Pólya-Szegö inequality for the Schwarz symmetrization, the minimum can be taken on radial and radially nonincreasing functions. Then they showed the existence of a minimizer $\hat{w} \in H^{1}\left(\mathbb{R}^{N}\right)$ by the direct method of the calculus of variations. With the Lagrange multiplier Theorem, they concluded that $\bar{u}(x):=\hat{w}\left(x / t_{\hat{w}}\right)$ with $t_{\hat{w}}=\sqrt{\frac{N-2}{2 N}}\|\nabla \hat{w}\|_{2}$ is a least energy solution of (1.3). By noting the oneto-one correspondence between $\mathcal{S}$ and $\mathcal{M}^{\infty}$, Jeanjean-Tanaka [6] proved that $\bar{u}$ is also a ground state solution of Pohožaev type for (1.3), i.e. $\bar{u} \in \mathcal{M}^{\infty}$ and satisfies

$$
I^{\infty}(\bar{u})=\inf _{\mathcal{M}^{\infty}} I^{\infty} .
$$

By using a different way, Shatah [12] showed that there exists $\tilde{u} \in \mathcal{M}_{r}^{\infty}$ such that

$$
I^{\infty}(\tilde{u})=\inf _{\mathcal{M}_{r}^{\infty}} I^{\infty},
$$

where

$$
\mathcal{M}_{r}^{\infty}:=\left\{u \in H_{r}^{1}\left(\mathbb{R}^{N}\right) \backslash\{0\}: \mathcal{P}^{\infty}(u)=0\right\}
$$


and

$$
H_{r}^{1}\left(\mathbb{R}^{N}\right)=\left\{u \in H^{1}\left(\mathbb{R}^{N}\right): u \text { is radially symmetric function on } \mathbb{R}^{N}\right\} .
$$

Obviously, (F1)-(F3) are satisfied by a very wide class of nonlinearities. In particular only conditions on $f(t)$ near $0, \infty$ and the point $s_{0}$ are required. Moreover, in view of [1, 2.2], (F1) is "almost" necessary, and (F2) and (F3) are necessary for the existence of a nontrivial solution of problem (1.3). For more related results under Berestycki-Lions conditions, we refer to [3, 4, 11, 18, 19].

When $V(x) / \equiv V_{\infty}$, the approach used in [1] does not work any more for nonautonomous equation (1.1), since the Schwarz symmetrization can only be applied to autonomous problems. In a different way, Rabinowitz [10] proved that (1.1) has a nontrivial solution if $V$ satisfies (V1) and (V2) and $f$ does (F1), (F2), the Nehari monotonic condition:

$$
f(t) /|t| \text { is strictly increasing on }(-\infty, 0) \cup(0, \infty) \text {; }
$$

and the global growth Ambrosetti-Rabinowitz condition:

$$
\text { there exists } \mu>2 \text { such that } f(t) t \geq \mu F(t)>0, \forall t \in \mathbb{R} \backslash\{0\} \text {. }
$$

$(\mathrm{Ne})$ and $(\mathrm{AR})$ are used to recover the compactness and to get the boundedness of Palais-Smale sequences, respectively, see [17] for more details. By means of Jeanjean's monotonicity trick, developed in [5], which is a generalization of the Struwe's one (see [13]), consisting in a suitable approximating method, Jeanjean and Tanaka [7] derived an existence result using two weaker conditions instead of (Ne) and (AR). More precisely, Jeanjean and Tanaka proved that (1.1) has a least energy solution if $f$ satisfies (F1), (F2) and the following nonnegativity condition:

$$
f(t) \geq 0 \text { for } t \geq 0 \text {; }
$$

and the superlinear growth condition:

$$
\lim _{t \rightarrow+\infty} \frac{f(t)}{t}=\infty ;
$$

and $V$ does (V1), (V2) and the decay condition:

$$
\begin{array}{r}
V \in \mathcal{C}^{1}\left(\mathbb{R}^{N}, \mathbb{R}\right) \text { and there exists } \varphi \in L^{2}\left(\mathbb{R}^{N}\right) \cap W^{1, \infty}\left(\mathbb{R}^{N}\right) \text { such that } \\
\qquad|\nabla V(x)||x| \leq[\varphi(x)]^{2}, \quad \forall x \in \mathbb{R}^{N} .
\end{array}
$$

Clearly, (NG) and (SL) are stronger than (F3), moreover, (Vd) puts relatively strict constrains on the decay of $|\nabla V(x)|$. For example, $V(x)=a-\frac{b}{1+\mid x \alpha^{\alpha}}$ does not satisfy (Vd) for $a, b>0$ and $0<\alpha \leq N$.

Motivated by the work of $[1,2,7,12,16]$, we shall develop a more direct approach (the least energy squeeze approach) to show that (1.1) has a solution $\bar{u} \in \mathcal{M}$ such that $I(\bar{u})=\inf _{\mathcal{M}} I$ under (F1)-(F3), (V1), (V2) and an additional decay condition on $V$ :

$$
V \in \mathcal{C}^{1}\left(\mathbb{R}^{N}, \mathbb{R}\right) \text { and there exists } \theta \in[0,1) \text { such that } t \mapsto N V(t x)+\nabla V(t x) \cdot(t x)+\frac{\theta(N-2)^{3}}{4 t^{2}|x|^{2}} \text { is nonin- }
$$
creasing on $(0, \infty)$ for all $x \in \mathbb{R}^{N} \backslash\{0\}$,

where

and

$$
\mathcal{M}:=\left\{u \in H^{1}\left(\mathbb{R}^{N}\right) \backslash\{0\}: \mathcal{P}(u)=0\right\}
$$

$$
\mathcal{P}(u):=\frac{N-2}{2}\|\nabla u\|_{2}^{2}+\frac{1}{2} \int_{\mathbb{R}^{N}}[N V(x)+\nabla V(x) \cdot x] u^{2} \mathrm{~d} x-N \int_{\mathbb{R}^{N}} F(u) \mathrm{d} x
$$


is the Pohožaev functional associated with (1.1) (see [7]).

To prove the above conclusion, we shall divide our arguments into three steps: i). Choosing a minimizing sequence $\left\{u_{n}\right\}$ of $I$ on $\mathcal{M}$, which satisfies

$$
I\left(u_{n}\right) \rightarrow m:=\inf _{\mathcal{M}} I, \quad \mathcal{P}\left(u_{n}\right)=0 .
$$

Then showing that $\left\{u_{n}\right\}$ is bounded in $H^{1}\left(\mathbb{R}^{N}\right)$. With a concentration-compactness argument, showing that $\left\{u_{n}\right\}$ converges to some $\bar{u} \in H^{1}\left(\mathbb{R}^{N}\right) \backslash\{0\}$ up to translations and extraction of a subsequence. ii). Showing that $\bar{u} \in \mathcal{M}$ and $I(\bar{u})=\inf _{\mathcal{M}} I$. iii). Showing that $\bar{u}$ is a critical point of $I$. Of them, Step ii) is the most difficult due to lack of global compactness and adequate information on $I^{\prime}\left(u_{n}\right)$. Since (1.1) is nonautonomous, the radial compactness does not work for $\mathcal{M}$. To overcome this difficulty, we establish a crucial inequality related to $I(u), I\left(u_{t}\right)$ and $\mathcal{P}(u)$ (the IIP inequality in short, see Lemma 2.2), where $u_{t}(x)=u(x / t)$, it plays an important role in many places of this paper. With the help of the IIP inequality, we then can complete Step ii) by using Lions' concentration compactness, the least energy squeeze approach and some subtle analysis. In particular, we only use Lions' concentration compactness in our arguments, the radial and other compactness are not required, see the proofs of Lemmas 2.12 and 2.13. Moreover, such an approach could be useful for the study of other problems where radial symmetry of bounded sequence either fails or is not readily available. In Step iii), usually, one uses the Lagrange multipliers Theorem to show that the minimizer $\bar{u}$ is a critical point of $I$, but it is impossible to verify $\mathcal{P}^{\prime}(u) \neq 0$ for all $u \in \mathcal{N}$ under (V1)-(V3) and (F1)-(F3). To overcome this difficulty, we employ the combination of the IIP inequality and a deformation lemma, see Lemma 2.14. We believe that our approach could be applied to deal with many similar problems, such as quasilinear Schrödinger equations, Choquard equations and fractional Schrödinger equations.

Remark 1.1. There are indeed functions which satisfy (V1)-(V3). An example is given by $V(x)=V_{1}-\frac{A}{|x|^{2}+1}$, where $V_{1} \geq A$ and $0<A<(N-2)^{3} / 2(2 N+1)$ are two positive constants.

We are now in a position to state the main results on ground state solutions of Pohožaev type.

Theorem 1.2. Assume that $V$ and $f$ satisfy (V1)-(V3) and (F1)-(F3). Then problem (1.1) has a solution $\bar{u} \in$ $H^{1}\left(\mathbb{R}^{N}\right)$ such that $I(\bar{u})=\inf _{\mathcal{M}} I=\inf _{u \in \Lambda} \max _{t>0} I\left(u_{t}\right)>0$, where

$$
u_{t}(x):=u(x / t) \text { and } \Lambda=\left\{u \in H^{1}\left(\mathbb{R}^{N}\right): \int_{\mathbb{R}^{N}}\left[\frac{1}{2} V_{\infty} u^{2}-F(u)\right] \mathrm{d} x<0\right\} \text {. }
$$

As a consequence of Theorem 1.2, we can prove the following theorem.

Theorem 1.3. Assume that $f$ satisfies (F1)-(F3). Then problem (1.3) has a solution $\bar{u} \in H^{1}\left(\mathbb{R}^{N}\right)$ such that $I^{\infty}(\bar{u})=$ $\inf _{\mathcal{M}^{\infty}} I^{\infty}=\inf _{u \in \Lambda} \max _{t>0} I^{\infty}\left(u_{t}\right)>0$.

Remark 1.4. We point out that, as a consequence of Theorem 1.2, the least energy value $m:=\inf _{\mathcal{M}} I$ has a minimax characterization $m=\inf _{u \in \Lambda} \max _{t>0} I\left(u_{t}\right)$ which is much simpler than the usual characterizations related to the Mountain Pass level.

In the second part of the paper, we are interested in the existence of the least energy solutions for (1.1) under (F1)-(F3). In this case, we can replace (V3) by the following weaker decay assumption on $\nabla V$ :

$$
\begin{aligned}
& V \in \mathcal{C}^{1}\left(\mathbb{R}^{N}, \mathbb{R}\right) \text { and there exists } \theta \in[0,1) \text { such that } \\
& \qquad \nabla V(x) \cdot x \leq \frac{(N-2)^{2} \theta}{2|x|^{2}}, \quad \forall x \in \mathbb{R}^{N} \backslash\{0\} .
\end{aligned}
$$


As in Jeanjean and Tanaka [6], for $\lambda \in[1 / 2,1]$ we consider a family of functionals $I_{\lambda}: H^{1}\left(\mathbb{R}^{N}\right) \rightarrow \mathbb{R}$ defined by

$$
I_{\lambda}(u)=\frac{1}{2} \int_{\mathbb{R}^{N}}\left(|\nabla u|^{2}+V(x) u^{2}\right) \mathrm{d} x-\lambda \int_{\mathbb{R}^{N}} F(u) \mathrm{d} x .
$$

These functionals have a Mountain Pass geometry, and denoting the corresponding Mountain Pass levels by $c_{\lambda}$. Corresponding to (1.14), we also let

$$
I_{\lambda}^{\infty}(u)=\frac{1}{2} \int_{\mathbb{R}^{N}}\left(|\nabla u|^{2}+V_{\infty} u^{2}\right) \mathrm{d} x-\lambda \int_{\mathbb{R}^{N}} F(u) \mathrm{d} x .
$$

By Theorem 1.3, for every $\lambda \in[1 / 2,1]$, there exists a minimizer $u_{\lambda}^{\infty}$ of $I_{\lambda}^{\infty}$ on $\mathcal{M}_{\lambda}^{\infty}$, where

$$
\mathcal{M}_{\lambda}^{\infty}:=\left\{u \in H^{1}\left(\mathbb{R}^{N}\right) \backslash\{0\}: \mathcal{P}_{\lambda}^{\infty}(u)=0\right\}
$$

and

$$
\mathcal{P}_{\lambda}^{\infty}(u)=\frac{N-2}{2}\|\nabla u\|_{2}^{2}+\frac{N V_{\infty}}{2}\|u\|_{2}^{2}-N \lambda \int_{\mathbb{R}^{N}} F(u) \mathrm{d} x .
$$

Let

$$
A(u)=\frac{1}{2} \int_{\mathbb{R}^{N}}\left(|\nabla u|^{2}+V(x) u^{2}\right) \mathrm{d} x, \quad B(u)=\frac{1}{2} \int_{\mathbb{R}^{N}} F(u) \mathrm{d} x .
$$

Then $I_{\lambda}(u)=A(u)-\lambda B(u)$. Since $B(u)$ is not sign definite, it prevents us from employing Jeanjean's monotonicity trick [5]. Thanks to the work of Jeanjean-Toland [8], $I_{\lambda}$ still has a bounded (PS)-sequence $\left\{u_{n}(\lambda)\right\} \subset H^{1}\left(\mathbb{R}^{N}\right)$ at level $c_{\lambda}$ for almost every $\lambda \in[1 / 2,1]$. Different from the arguments in the existing literature, by means of $u_{1}^{\infty}$ and the IIP inequality, we can find $\bar{\lambda} \in[1 / 2,1)$ and then directly prove the following crucial inequality

$$
c_{\lambda}<m_{\lambda}^{\infty}:=\inf _{\mathcal{M}_{\lambda}^{\infty}} I_{\lambda}^{\infty}, \quad \lambda \in(\bar{\lambda}, 1],
$$

which is used to recover the compactness to (PS)-sequence $\left\{u_{n}(\lambda)\right\}$, see Lemmas 3.5 and 3.7. In particular, it is not required any information on sign of $u_{1}^{\infty}$ in our arguments. Applying (1.18) and a precise decomposition of bounded (PS)-sequence in [6], we can get a nontrivial critical point $u_{\lambda}$ of $I_{\lambda}$ which possesses energy $c_{\lambda}$ for almost every $\lambda \in(\bar{\lambda}, 1]$. Finally, with a Pohožaev identity we proved that (1.1) admits a least energy solution under (V1), (V2), (V4) and (F1)-(F3). More precisely, we have the following theorem.

Theorem 1.5. Assume that $V$ and $f$ satisfy (V1), (V2), (V4) and (F1)-(F3). Then problem (1.1) has a least energy solution.

Remark 1.6. Relative to (Vd), there seem to be more functions satisfying (V4). For example, it is easy to verify that $V(x)=a-\frac{b}{1+|x|^{\alpha}}$ satisfies (V4) for $\alpha \geq 2, a>0$ and

$$
4 b<(N-2)^{2} \text { if } \alpha=2 ; \quad \frac{(\alpha-2)^{(\alpha-2) / \alpha}(\alpha+2)^{(\alpha+2) / \alpha}}{2 \alpha} b<(N-2)^{2}, \text { if } \alpha>2 .
$$

However, it does not satisfy (Vd) when $2 \leq \alpha \leq N$.

Applying Theorem 1.5 to the following perturbed problem:

$$
\left\{\begin{array}{l}
-\triangle u+\left[V_{\infty}-\varepsilon h(x)\right] u=f(u), \quad x \in \mathbb{R}^{N} ; \\
u \in H^{1}\left(\mathbb{R}^{N}\right),
\end{array}\right.
$$

where $V_{\infty}$ is a positive constant and the function $h \in \mathcal{C}^{1}\left(\mathbb{R}^{N}, \mathbb{R}\right)$ verifies:

(H1) $h(x) \geq 0$ for all $x \in \mathbb{R}^{N}$ and $\lim _{|x| \rightarrow \infty} h(x)=0$;

(H2) $\sup _{x \in \mathbb{R}^{N}}\left[-|x|^{2}(\nabla h(x), x)\right]<\infty$.

Then we have the following corollary. 
Corollary 1.7. Assume that $h$ and $f$ satisfy (H1), (H2) and (F1)-(F3). Then there exists a constant $\varepsilon_{0}>0$ such that problem (1.19) has a least energy solution $\bar{u}_{\varepsilon} \in H^{1}\left(\mathbb{R}^{N}\right) \backslash\{0\}$ for all $0<\varepsilon \leq \varepsilon_{0}$.

Classically, in order to show the existence of solutions for (1.1), one compares the critical level of $I$ with the one of $I^{\infty}$ (i.e. the energy functional corresponds to the problem at infinity). To this end, it is necessary to establish a strict inequality similar to

$$
\max _{t \in[0,1]} I\left(y_{0}(t)\right)<\inf \left\{I^{\infty}(u): u \in H^{1}\left(\mathbb{R}^{N}\right) \backslash\{0\} \text { is a solution of }(1.3)\right\}
$$

for some path $y_{0} \in \mathcal{C}\left([0,1], H^{1}\left(\mathbb{R}^{N}\right)\right)$. Clearly, $y_{0}(t)>0$ is a natural requirement under (V1) and (V2). But we only need $y_{0}(t) \neq 0$ in our arguments. Therefore, our approach could be useful for the study of other problems where paths or the ground state solutions of the problem at infinity are not sign definite.

Throughout the paper we make use of the following notations:

a $H^{1}\left(\mathbb{R}^{N}\right)$ denotes the usual Sobolev space equipped with the inner product and norm

$$
(u, v)=\int_{\mathbb{R}^{N}}(\nabla u \cdot \nabla v+u v) \mathrm{d} x,\|u\|=(u, u)^{1 / 2}, \forall u, v \in H^{1}\left(\mathbb{R}^{N}\right) ;
$$

A $L^{s}\left(\mathbb{R}^{N}\right)(1 \leq s<\infty)$ denotes the Lebesgue space with the norm $\|u\|_{s}=\left(\int_{\mathbb{R}^{N}}|u|^{s} \mathrm{~d} x\right)^{1 / s}$;

A. For any $u \in H^{1}\left(\mathbb{R}^{N}\right) \backslash\{0\}, u_{t}(x):=u(x / t)$ for $t>0$;

A For any $x \in \mathbb{R}^{N}$ and $r>0, B_{r}(x):=\left\{y \in \mathbb{R}^{N}:|y-x|<r\right\}$;

a $C_{1}, C_{2}, \cdots$ denote positive constants possibly different in different places.

The rest of the paper is organized as follows. In Section 2, we give some preliminaries, and give the proof of Theorem 1.2. Section 3 is devoted to finding a least energy solution for (1.1) and Theorem 1.5 will be proved in this section.

\section{Ground state solutions for (1.1)}

In this section, we give the proofs of Theorem 1.2. To this end, we give some useful lemmas. Since $V(x) \equiv$ $V_{\infty}$ satisfies (V1)-(V3), thus all conclusions on $I$ are also true for $I^{\infty}$. For (1.3), we always assume that $V_{\infty}>0$. By a simple calculation, we can verify Lemma 2.1.

Lemma 2.1. The following inequality holds:

$$
\mathfrak{g}(t):=2-N t^{N-2}+(N-2) t^{N}>\mathfrak{g}(1)=0, \quad \forall t \in[0,1) \cup(1,+\infty) .
$$

Moreover (V3) implies the following inequality holds:

$$
\begin{aligned}
& N t^{N}[V(x)-V(t x)]+\left(t^{N}-1\right) \nabla V(x) \cdot x \\
\geq & -\frac{(N-2)^{2} \theta\left[2-N t^{N-2}+(N-2) t^{N}\right]}{4|x|^{2}}, \quad \forall t \geq 0, \quad x \in \mathbb{R}^{N} \backslash\{0\} .
\end{aligned}
$$

Lemma 2.2. Assume that (V1), (V3), (F1) and (F2) hold. Then

$$
\begin{aligned}
I(u) \geq I\left(u_{t}\right)+\frac{1-t^{N}}{N} \mathcal{P}(u)+\frac{(1-\theta)\left[2-N t^{N-2}+(N-2) t^{N}\right]}{2 N}\|\nabla u\|_{2}^{2}, \\
\forall u \in H^{1}\left(\mathbb{R}^{N}\right), \quad t>0 .
\end{aligned}
$$


Proof. According to Hardy inequality, we have

$$
\|\nabla u\|_{2}^{2} \geq \frac{(N-2)^{2}}{4} \int_{\mathbb{R}^{N}} \frac{u^{2}}{|x|^{2}} \mathrm{~d} x, \forall u \in H^{1}\left(\mathbb{R}^{N}\right) .
$$

Note that

$$
I\left(u_{t}\right)=\frac{t^{N-2}}{2}\|\nabla u\|_{2}^{2}+\frac{t^{N}}{2} \int_{\mathbb{R}^{N}} V(t x) u^{2} \mathrm{~d} x-t^{N} \int_{\mathbb{R}^{N}} F(u) \mathrm{d} x .
$$

Thus, by (1.2), (1.12), (2.1), (2.2), (2.4) and (2.5), one has

$$
\begin{aligned}
I(u)-I\left(u_{t}\right)= & \frac{1-t^{N-2}}{2}\|\nabla u\|_{2}^{2}+\frac{1}{2} \int_{\mathbb{R}^{N}}\left[V(x)-t^{N} V(t x)\right] u^{2} \mathrm{~d} x-\left(1-t^{N}\right) \int_{\mathbb{R}^{N}} F(u) \mathrm{d} x \\
= & \frac{1-t^{N}}{N}\left\{\frac{N-2}{2}\|\nabla u\|_{2}^{2}+\frac{1}{2} \int_{\mathbb{R}^{N}}[N V(x)+\nabla V(x) \cdot x] u^{2} \mathrm{~d} x-N \int_{\mathbb{R}^{N}} F(u) \mathrm{d} x\right\} \\
& +\frac{2-N t^{N-2}+(N-2) t^{N}}{2 N}\|\nabla u\|_{2}^{2}+\frac{1}{2} \int_{\mathbb{R}^{N}}\left\{t^{N}[V(x)-V(t x)]-\frac{1-t^{N}}{N} \nabla V(x) \cdot x\right\} u^{2} \mathrm{~d} x \\
\geq & \frac{1-t^{N}}{N} \mathcal{P}(u)+\frac{(1-\theta)\left[2-N t^{N-2}+(N-2) t^{N}\right]}{2 N}\|\nabla u\|_{2}^{2}, \quad \forall u \in H^{1}\left(\mathbb{R}^{N}\right), \quad t>0 .
\end{aligned}
$$

This shows that (2.3) holds.

From Lemma 2.2, we have the following two corollaries.

Corollary 2.3. Assume that (F1) and (F2) hold. Then

$$
\begin{gathered}
I^{\infty}(u)=I^{\infty}\left(u_{t}\right)+\frac{1-t^{N}}{N} \mathcal{P}^{\infty}(u)+\frac{2-N t^{N-2}+(N-2) t^{N}}{2 N}\|\nabla u\|_{2}^{2}, \\
\forall u \in H^{1}\left(\mathbb{R}^{N}\right), \quad t>0 .
\end{gathered}
$$

Corollary 2.4. Assume that (V1), (V3), (F1) and (F2) hold. Then for $u \in \mathcal{M}$

$$
I(u)=\max _{t>0} I\left(u_{t}\right) .
$$

Lemma 2.5. Assume that (V1)-(V3) hold. Then there exist two constants $y_{1}, y_{2}>0$ such that

$$
y_{1}\|u\|^{2} \leq(N-2)\|\nabla u\|_{2}^{2}+\int_{\mathbb{R}^{N}}[N V(x)+\nabla V(x) \cdot x] u^{2} \mathrm{~d} x \leq y_{2}\|u\|^{2}, \quad \forall u \in H^{1}\left(\mathbb{R}^{N}\right) .
$$

Proof. Let $t=0$ and $t \rightarrow \infty$ in (2.2), respectively, and using (V2), one has

$$
-\frac{(N-2)^{3} \theta}{4|x|^{2}}+N V_{\infty} \leq N V(x)+\nabla V(x) \cdot x \leq N V_{\infty}+\frac{(N-2)^{2} \theta}{2|x|^{2}}, \quad \forall x \in \mathbb{R}^{N} \backslash\{0\} .
$$

Thus it follows from (2.4) and (2.9) that

$$
\begin{aligned}
(N-2)\|\nabla u\|_{2}^{2}+\int_{\mathbb{R}^{N}}[N V(x)+\nabla V(x) \cdot x] u^{2} \mathrm{~d} x & \leq(N-2+2 \theta)\|\nabla u\|_{2}^{2}+N V_{\infty}\|u\|_{2}^{2} \\
& \leq\left[N-2+2 \theta+N V_{\infty}\right]\|u\|^{2}:=y_{2}\|u\|^{2}, \quad \forall u \in H^{1}\left(\mathbb{R}^{N}\right)
\end{aligned}
$$


and

$$
\begin{aligned}
& (N-2)\|\nabla u\|_{2}^{2}+\int_{\mathbb{R}^{N}}[N V(x)+\nabla V(x) \cdot x] u^{2} \mathrm{~d} x \\
\geq & (1-\theta)(N-2)\|\nabla u\|_{2}^{2}+N V_{\infty}\|u\|_{2}^{2} \\
\geq & \min \left\{(1-\theta)(N-2), N V_{\infty}\right\}\|u\|^{2}:=y_{1}\|u\|^{2}, \forall u \in H^{1}\left(\mathbb{R}^{N}\right) .
\end{aligned}
$$

Both (2.10) and (2.11) imply that (2.8) holds.

To show $\mathcal{M} \neq \emptyset$, we define a set $\Lambda$ as follows:

$$
\Lambda=\left\{u \in H^{1}\left(\mathbb{R}^{N}\right): \int_{\mathbb{R}^{N}}\left[\frac{1}{2} V_{\infty} u^{2}-F(u)\right] \mathrm{d} x<0\right\} .
$$

Lemma 2.6. Assume that (V1)-(V3) and (F1)-(F3) hold. Then $\Lambda \neq \emptyset$ and

$$
\left\{u \in H^{1}\left(\mathbb{R}^{N}\right) \backslash\{0\}: \mathcal{P}^{\infty}(u) \leq 0 \text { or } \mathcal{P}(u) \leq 0\right\} \subset \Lambda .
$$

Proof. In view of the proof of [1, Theorem 2], (F3) implies $\Lambda \neq \emptyset$. Next, we have two cases to distinguish:

1). $u \in H^{1}\left(\mathbb{R}^{N}\right) \backslash\{0\}$ and $\mathcal{P}^{\infty}(u) \leq 0$, then (1.5) implies $u \in \Lambda$.

2). $u \in H^{1}\left(\mathbb{R}^{N}\right) \backslash\{0\}$ and $\mathcal{P}(u) \leq 0$, then it follows from (1.12), (2.4) and (2.9) that

$$
\begin{aligned}
N \int_{\mathbb{R}^{N}}\left[\frac{1}{2} V_{\infty} u^{2}-F(u)\right] \mathrm{d} x & =\mathcal{P}(u)-\frac{N-2}{2}\|\nabla u\|_{2}^{2}-\frac{N}{2} \int_{\mathbb{R}^{N}}\left[\left(V(x)-V_{\infty}\right)+\frac{\nabla V(x) \cdot x}{N}\right] u^{2} \mathrm{~d} x \\
& \leq-\frac{N-2}{2}\|\nabla u\|_{2}^{2}+\frac{(N-2)^{3} \theta}{8} \int_{\mathbb{R}^{N}} \frac{u^{2}}{|x|^{2}} \mathrm{~d} x \\
& \leq-\frac{(1-\theta)(N-2)}{2}\|\nabla u\|_{2}^{2}<0,
\end{aligned}
$$

which implies $u \in \Lambda$.

Lemma 2.7. Assume that (V1)-(V3) and (F1)-(F3) hold. Then for any $u \in \Lambda$, there exists a unique $t_{u}>0$ such that $u_{t_{u}} \in \mathcal{M}$.

Proof. Let $u \in \Lambda$ be fixed and define a function $\zeta(t):=I\left(u_{t}\right)$ on $(0, \infty)$. Clearly, by (1.12) and (2.5), we have

$$
\begin{aligned}
& \zeta^{\prime}(t)=0 \\
& \Leftrightarrow \frac{N-2}{2} t^{N-2}\|\nabla u\|_{2}^{2}+\frac{t^{N}}{2} \int_{\mathbb{R}^{N}}[N V(t x)+\nabla V(t x) \cdot(t x)] u^{2} \mathrm{~d} x-N t^{N} \int_{\mathbb{R}^{N}} F(u) \mathrm{d} x=0 \\
& \Leftrightarrow \mathcal{P}\left(u_{t}\right)=0 \Leftrightarrow u_{t} \in \mathcal{M} .
\end{aligned}
$$

It is easy to verify, using (V1), (V2), (F1), (2.5) and the definition of $\Lambda$, that $\lim _{t \rightarrow 0} \zeta(t)=0, \zeta(t)>0$ for $t>0$ small and $\zeta(t)<0$ for $t$ large. Therefore $\max _{t \in[0, \infty)} \zeta(t)$ is achieved at some $t_{u}>0$ so that $\zeta^{\prime}\left(t_{u}\right)=0$ and $u_{t_{u}} \in \mathcal{M}$.

Next we claim that $t_{u}$ is unique for any $u \in \Lambda$. In fact, for any given $u \in \Lambda$, let $t_{1}, t_{2}>0$ such that $u_{t_{1}}, u_{t_{2}} \in \mathcal{M}$. Then $\mathcal{P}\left(u_{t_{1}}\right)=\mathcal{P}\left(u_{t_{2}}\right)=0$. Jointly with (2.3), we have

$$
\begin{aligned}
I\left(u_{t_{1}}\right) & \geq I\left(u_{t_{2}}\right)+\frac{t_{1}^{N}-t_{2}^{N}}{N t_{1}^{N}} \mathcal{P}\left(u_{t_{1}}\right)+\frac{(1-\theta)\left[2 t_{1}^{N}-N t_{1}^{2} t_{2}^{N-2}+(N-2) t_{2}^{N}\right]}{2 N t_{1}^{N}}\left\|\nabla u_{t_{1}}\right\|_{2}^{2} \\
& =I\left(u_{t_{2}}\right)+\frac{(1-\theta)\left[2 t_{1}^{N}-N t_{1}^{2} t_{2}^{N-2}+(N-2) t_{2}^{N}\right]}{2 N t_{1}^{2}}\|\nabla u\|_{2}^{2}
\end{aligned}
$$


and

$$
\begin{aligned}
I\left(u_{t_{2}}\right) & \geq I\left(u_{t_{1}}\right)+\frac{t_{2}^{N}-t_{1}^{N}}{N t_{2}^{N}} \mathcal{P}\left(u_{t_{2}}\right)+\frac{(1-\theta)\left[2 t_{2}^{N}-N t_{2}^{2} t_{1}^{N-2}+(N-2) t_{1}^{N}\right]}{2 N t_{2}^{N}}\left\|\nabla u_{t_{2}}\right\|_{2}^{2} \\
& =I\left(u_{t_{1}}\right)+\frac{(1-\theta)\left[2 t_{2}^{N}-N t_{2}^{2} t_{1}^{N-2}+(N-2) t_{1}^{N}\right]}{2 N t_{2}^{2}}\|\nabla u\|_{2}^{2} .
\end{aligned}
$$

(2.15) and (2.16) imply $t_{1}=t_{2}$. Therefore, $t_{u}>0$ is unique for any $u \in \Lambda$.

Corollary 2.8. Assume that (F1)-(F3) hold. Then for any $u \in \Lambda$, there exists a unique $t_{u}>0$ such that $u_{t_{u}} \in \mathcal{M}^{\infty}$.

From Corollary 2.4, Lemma 2.6 and Lemma 2.7, we have $\mathcal{M} \neq \emptyset$ and the following lemma.

Lemma 2.9. Assume that (V1)-(V3) and (F1)-(F3) hold. Then

$$
\inf _{u \in \mathcal{M}} I(u):=m=\inf _{u \in \Lambda} \max _{t>0} I\left(u_{t}\right) .
$$

The following lemma is a known result which can be proved by a standard argument (see $[14,15])$.

Lemma 2.10. Assume that (V1), (V2), (F1) and (F2) hold. If $u_{n} \rightarrow \bar{u}$ in $H^{1}\left(\mathbb{R}^{N}\right)$, then

$$
I\left(u_{n}\right)=I(\bar{u})+I\left(u_{n}-\bar{u}\right)+o(1)
$$

and

$$
\mathcal{P}\left(u_{n}\right)=\mathcal{P}(\bar{u})+\mathcal{P}\left(u_{n}-\bar{u}\right)+o(1) .
$$

Lemma 2.11. Assume that (V1)-(V3) and (F1)-(F3) hold. Then

(i) there exists $\rho_{0}>0$ such that $\|u\| \geq \rho_{0}, \forall u \in \mathcal{M}$;

(ii) $\quad m=\inf _{u \in \mathcal{M}} I(u)>0$.

Proof. i). Since $\mathcal{P}(u)=0, \forall u \in \mathcal{M}$, by (F1), (F2), (1.12), (2.8) and Sobolev embedding theorem, one has

$$
\begin{aligned}
\frac{y_{1}}{2}\|u\|^{2} & \leq \frac{N-2}{2}\|\nabla u\|_{2}^{2}+\frac{1}{2} \int_{\mathbb{R}^{N}}[N V(x)+\nabla V(x) \cdot x] u^{2} \mathrm{~d} x \\
& =N \int_{\mathbb{R}^{N}} F(u) \mathrm{d} x \\
& \leq \frac{y_{1}}{4}\|u\|^{2}+C_{1}\|u\|^{2^{*}}
\end{aligned}
$$

which implies

$$
\|u\| \geq \rho_{0}:=\left(\frac{y_{1}}{4 C_{1}}\right)^{(N-2) / 4}, \quad \forall u \in \mathcal{M} .
$$

ii). For $u \in H^{1}\left(\mathbb{R}^{N}\right)$, by the Sobolev inequality, one has $S\|u\|_{2^{*}}^{2} \leq\|\nabla u\|_{2}^{2}$. By (V2), there exists $R>0$ such that $V(x) \geq \frac{V_{\infty}}{2}$ for $|x| \geq R$. It follows from (F1) and (F2) that there exists $C_{2}>0$ such that

$$
|F(t)| \leq \frac{1}{4} \min \left\{\frac{S}{R^{2} \omega_{N}^{2 / N}}, V_{\infty}\right\}|t|^{2}+C_{2}|t|^{2^{*}}, \quad \forall t \in \mathbb{R},
$$

where $\omega_{N}$ denote the volume of the unit ball of $\mathbb{R}^{N}$. For $u \in \mathcal{M}$, let

$$
t_{u}=\left[\frac{(N-2) S^{N /(N-2)}}{4 N C_{2}}\right]^{1 / 2}\|\nabla u\|_{2}^{-2 /(N-2)} .
$$


Making use of the Hölder inequality and the Sobolev embedding theorem, we get

$$
\int_{\left|t_{u} x\right|<R} u^{2} \mathrm{~d} x \leq\left(\frac{\omega_{N} R^{N}}{t_{u}^{N}}\right)^{\left(2^{*}-2\right) / 2^{*}}\left(\int_{\left|t_{u} x\right|<R} u^{2^{*}} \mathrm{~d} x\right)^{2 / 2^{*}} \leq\left(\frac{\omega_{N} R^{N}}{t_{u}^{N}}\right)^{2 / N} S^{-1}\|\nabla u\|_{2}^{2} .
$$

Then from (2.3), (2.5), (2.21), (2.22) and the Sobolev embedding theorem, we have

$$
\begin{aligned}
I(u) \geq & I\left(u_{t_{u}}\right) \\
= & \frac{t_{u}^{N-2}}{2}\|\nabla u\|_{2}^{2}+\frac{t_{u}^{N}}{2} \int_{\mathbb{R}^{N}} V\left(t_{u} x\right) u^{2} \mathrm{~d} x-t_{u}^{N} \int_{\mathbb{R}^{N}} F(u) \mathrm{d} x \\
\geq & \frac{t_{u}^{N-2}}{4}\|\nabla u\|_{2}^{2}+\frac{S}{4 R^{2} \omega_{N}^{2 / N}} t_{u}^{N} \int_{\left|t_{u} x\right|<R} u^{2} \mathrm{~d} x+\frac{V_{\infty} t_{u}^{N}}{4} \int_{\left|t_{u} x\right| \geq R} u^{2} \mathrm{~d} x \\
& -t_{u}^{N} \int_{\mathbb{R}^{N}} F(u) \mathrm{d} x \\
\geq & \frac{t_{u}^{N-2}}{4}\|\nabla u\|_{2}^{2}+\frac{1}{4} \min \left\{\frac{S}{R^{2} \omega_{N}^{2 / N}}, V_{\infty}\right\} t_{u}^{N}\|u\|_{2}^{2}-t_{u}^{N} \int_{\mathbb{R}^{N}} F(u) \mathrm{d} x \\
\geq & \frac{t_{u}^{N-2}}{4}\|\nabla u\|_{2}^{2}-C_{2} t_{u}^{N}\|u\|_{2^{*}}^{2^{*}} \\
\geq & \frac{t_{u}^{N-2}}{4}\|\nabla u\|_{2}^{2}-C_{2} S^{-N /(N-2)} t_{u}^{N}\|\nabla u\|_{2}^{2 N /(N-2)} \\
= & \frac{S^{N / 2}}{2^{N-1}(N-2) C_{2}^{(N-2) / 2}}\left(\frac{N-2}{N}\right)^{N / 2}, \quad \forall u \in \mathcal{M} .
\end{aligned}
$$

This shows that $m=\inf _{u \in \mathcal{M}} I(u)>0$.

Lemma 2.12. Assume that (V1)-(V3) and (F1)-(F3) hold. Then $m \leq m^{\infty}=\inf _{u \in \mathcal{M}^{\infty}} I^{\infty}(u)$.

Proof. Arguing indirectly, we assume that $m>m^{\infty}$. Let $\varepsilon:=m-m^{\infty}$. Then there exists $u_{\varepsilon}^{\infty}$ such that

$$
u_{\varepsilon}^{\infty} \in \mathcal{M}^{\infty} \text { and } m^{\infty}+\frac{\varepsilon}{2}>I^{\infty}\left(u_{\varepsilon}^{\infty}\right)
$$

In view of Lemmas 2.6 and 2.7, there exists $t_{\varepsilon}>0$ such that $\left(u_{\varepsilon}^{\infty}\right)_{t_{\varepsilon}} \in \mathcal{M}$. Thus, it follows from (V1), (V2), (1.2), (1.4), (2.6) and (2.23) that

$$
m^{\infty} \frac{\varepsilon}{2}>I^{\infty}\left(u_{\varepsilon}^{\infty}\right) \geq I^{\infty}\left(\left(u_{\varepsilon}^{\infty}\right)_{t_{\varepsilon}}\right) \geq I\left(\left(u_{\varepsilon}^{\infty}\right)_{t_{\varepsilon}}\right) \geq m .
$$

This contradiction shows the conclusion of Lemma 2.12 is true.

Lemma 2.13. Assume that (V1)-(V3) and (F1)-(F3) hold. Then $m$ is achieved.

Proof. In view of Lemmas 2.6, 2.7 and 2.11, we have $\mathcal{M} \neq \emptyset$ and $m>0$. Let $\left\{u_{n}\right\} \subset \mathcal{M}$ be such that $I\left(u_{n}\right) \rightarrow m$. Since $\mathcal{P}\left(u_{n}\right)=0$, then it follows from (2.3) with $t \rightarrow 0$ that

$$
m+o(1)=I\left(u_{n}\right) \geq \frac{1-\theta}{N}\left\|\nabla u_{n}\right\|_{2}^{2} .
$$

This shows that $\left\{\left\|\nabla u_{n}\right\|_{2}\right\}$ is bounded. Next, we prove that $\left\{\left\|u_{n}\right\|\right\}$ is also bounded. By (F1), (F2), (1.12), (2.8) and the Sobolev embedding theorem, one has

$$
\begin{aligned}
y_{1}\left\|u_{n}\right\|^{2} & \leq(N-2)\left\|\nabla u_{n}\right\|_{2}^{2}+\int_{\mathbb{R}^{N}}[N V(x)+\nabla V(x) \cdot x] u_{n}^{2} \mathrm{~d} x \\
& =2 N \int_{\mathbb{R}^{N}} F\left(u_{n}\right) \mathrm{d} x
\end{aligned}
$$




$$
\begin{aligned}
& \leq \frac{y_{1}}{2}\left\|u_{n}\right\|^{2}+C_{4}\left\|u_{n}\right\|_{2^{2^{*}}}^{2^{*}} \\
& \leq \frac{y_{1}}{2}\left\|u_{n}\right\|^{2}+C_{4} S^{-2^{*} / 2}\left\|\nabla u_{n}\right\|_{2}^{2^{*}} .
\end{aligned}
$$

This shows that $\left\{u_{n}\right\}$ is bounded in $H^{1}\left(\mathbb{R}^{N}\right)$. Passing to a subsequence, we have $u_{n} \rightarrow \bar{u}$ in $H^{1}\left(\mathbb{R}^{N}\right)$. Then $u_{n} \rightarrow \bar{u}$ in $L_{\text {loc }}^{s}\left(\mathbb{R}^{N}\right)$ for $2 \leq s<2^{\star}$ and $u_{n} \rightarrow \bar{u}$ a.e. in $\mathbb{R}^{N}$. There are two possible cases: i). $\bar{u}=0$ and ii). $\bar{u} \neq 0$.

Case i). $\bar{u}=0$, i.e. $u_{n} \rightarrow 0$ in $H^{1}\left(\mathbb{R}^{N}\right)$. Then $u_{n} \rightarrow 0$ in $L_{\text {loc }}^{s}\left(\mathbb{R}^{N}\right)$ for $2 \leq s<2^{\star}$ and $u_{n} \rightarrow 0$ a.e. in $\mathbb{R}^{N}$. By (V2) and (2.9), it is easy to show that

$$
\lim _{n \rightarrow \infty} \int_{\mathbb{R}^{N}}\left[V_{\infty}-V(x)\right] u_{n}^{2} \mathrm{~d} x=\lim _{n \rightarrow \infty} \int_{\mathbb{R}^{N}} \nabla V(x) \cdot x u_{n}^{2} \mathrm{~d} x=0 .
$$

From (1.2), (1.4), (1.5), (1.12) and (2.26), one can get

$$
I^{\infty}\left(u_{n}\right) \rightarrow m, \quad \mathcal{P}^{\infty}\left(u_{n}\right) \rightarrow 0
$$

From Lemma 2.11 (i), (1.5) and (2.27), one has

$$
\begin{aligned}
\min \left\{N-2, N V_{\infty}\right\} \rho_{0}^{2} & \leq \min \left\{N-2, N V_{\infty}\right\}\left\|u_{n}\right\|^{2} \\
& \leq(N-2)\left\|\nabla u_{n}\right\|_{2}^{2}+N V_{\infty}\left\|u_{n}\right\|_{2}^{2} \\
& =2 N \int_{\mathbb{R}^{N}} F\left(u_{n}\right) \mathrm{d} x+o(1) .
\end{aligned}
$$

Using (F1), (F2), (2.28) and Lions' concentration compactness principle [20, Lemma 1.21], we can prove that there exist $\delta>0$ and a sequence $\left\{y_{n}\right\} \subset \mathbb{R}^{N}$ such that $\int_{B_{1}\left(y_{n}\right)}\left|u_{n}\right|^{2} \mathrm{~d} x>\delta$. Let $\hat{u}_{n}(x)=u_{n}\left(x+y_{n}\right)$. Then we have $\left\|\hat{u}_{n}\right\|=\left\|u_{n}\right\|$ and

$$
\mathcal{P}^{\infty}\left(\hat{u}_{n}\right)=o(1), \quad I^{\infty}\left(\hat{u}_{n}\right) \rightarrow m, \quad \int_{B_{1}(0)}\left|\hat{u}_{n}\right|^{2} \mathrm{~d} x>\delta .
$$

Therefore, there exists $\hat{u} \in H^{1}\left(\mathbb{R}^{N}\right) \backslash\{0\}$ such that, passing to a subsequence,

$$
\begin{cases}\hat{u}_{n} \rightarrow \hat{u}, & \text { in } H^{1}\left(\mathbb{R}^{N}\right) ; \\ \hat{u}_{n} \rightarrow \hat{u}, & \text { in } L_{\text {loc }}^{S}\left(\mathbb{R}^{N}\right), \forall s \in\left[1,2^{\star}\right) ; \\ \hat{u}_{n} \rightarrow \hat{u}, & \text { a.e. on } \mathbb{R}^{N} .\end{cases}
$$

Let $w_{n}=\hat{u}_{n}-\hat{u}$. Then (2.30) and Lemma 2.10 yield

$$
I^{\infty}\left(\hat{u}_{n}\right)=I^{\infty}(\hat{u})+I^{\infty}\left(w_{n}\right)+o(1)
$$

and

$$
\mathcal{P}^{\infty}\left(\hat{u}_{n}\right)=\mathcal{P}^{\infty}(\hat{u})+\mathcal{P}^{\infty}\left(w_{n}\right)+o(1) .
$$

Moreover,

$$
\frac{1}{N}\left\|\nabla w_{n}\right\|_{2}^{2}=m-\frac{1}{N}\|\nabla \hat{u}\|_{2}^{2}+o(1), \quad \mathcal{P}^{\infty}\left(w_{n}\right)=-\mathcal{P}^{\infty}(\hat{u})+o(1) .
$$

If there exists a subsequence $\left\{w_{n_{i}}\right\}$ of $\left\{w_{n}\right\}$ such that $w_{n_{i}}=0$, then going to this subsequence, we have

$$
I^{\infty}(\hat{u})=m, \quad \mathcal{P}^{\infty}(\hat{u})=0 .
$$

Next, we assume that $w_{n} \neq 0$. We claim that $\mathcal{P}^{\infty}(\hat{u}) \leq 0$. Otherwise, if $\mathcal{P}^{\infty}(\hat{u})>0$, then (2.33) implies $\mathcal{P}^{\infty}\left(w_{n}\right)<0$ for large $n$. In view of Lemma 2.6 and Corollary 2.8, there exists $t_{n}>0$ such that $\left(w_{n}\right)_{t_{n}} \in \mathcal{M}^{\infty}$. From (1.4), (1.5), (2.6) and (2.33), we obtain

$$
\begin{aligned}
m-\frac{1}{N}\|\nabla \hat{u}\|_{2}^{2}+o(1) & =\frac{1}{N}\left\|\nabla w_{n}\right\|_{2}^{2} \\
& =I^{\infty}\left(w_{n}\right)-\frac{1}{N} \mathcal{P}^{\infty}\left(w_{n}\right)
\end{aligned}
$$




$$
\begin{aligned}
& \geq \quad I^{\infty}\left(\left(w_{n}\right)_{t_{n}}\right)-\frac{t_{n}^{N}}{N} \mathcal{P}^{\infty}\left(w_{n}\right) \\
& \geq \quad m^{\infty}-\frac{t_{n}^{N}}{N} \mathcal{P}^{\infty}\left(w_{n}\right) \geq m^{\infty},
\end{aligned}
$$

which implies $\mathcal{P}^{\infty}(\hat{u}) \leq 0$ due to $\|\nabla \hat{u}\|_{2}>0$. Since $\hat{u} \neq 0$ and $\mathcal{P}^{\infty}(\hat{u}) \leq 0$, in view of Lemma 2.6 and Corollary 2.8, there exists $\hat{t}>0$ such that $\hat{u}_{\hat{t}} \in \mathcal{N}^{\infty}$. From (1.4), (1.5), (2.6), (2.29) and the weak semicontinuity of norm, one has

$$
\begin{aligned}
m & =\lim _{n \rightarrow \infty}\left[I^{\infty}\left(\hat{u}_{n}\right)-\frac{1}{N} \mathcal{P}^{\infty}\left(\hat{u}_{n}\right)\right] \\
& =\frac{1}{N} \lim _{n \rightarrow \infty}\left\|\nabla \hat{u}_{n}\right\|_{2}^{2} \geq \frac{1}{N}\|\nabla \hat{u}\|_{2}^{2} \\
& =I^{\infty}(\hat{u})-\frac{1}{N} \mathcal{P}^{\infty}(\hat{u}) \geq I^{\infty}\left(\hat{u}_{\hat{t}}\right)-\frac{\hat{t}^{N}}{N} \mathcal{P}^{\infty}(\hat{u}) \\
& \geq m^{\infty}-\frac{\hat{t}^{N}}{N} \mathcal{P}^{\infty}(\hat{u}) \\
& \geq m-\frac{\hat{t}^{N}}{N} \mathcal{P}^{\infty}(\hat{u}) \geq m,
\end{aligned}
$$

which implies (2.34) holds also. In view of Lemmas 2.6 and 2.7, there exists $\tilde{t}>0$ such that $\hat{u}_{\tilde{t}} \in \mathcal{M}$, moreover, it follows from (V2), (1.2), (1.4), (2.34) and Corollary 2.3 that

$$
m \leq I\left(\hat{u}_{\tilde{t}}\right) \leq I^{\infty}\left(\hat{u}_{\tilde{t}}\right) \leq I^{\infty}(\hat{u})=m .
$$

This shows that $m$ is achieved at $\hat{u}_{\tilde{t}} \in \mathcal{N}$.

Case ii). $\bar{u} \neq 0$. Let $v_{n}=u_{n}-\bar{u}$. Then Lemma 2.10 yields

$$
I\left(u_{n}\right)=I(\bar{u})+I\left(v_{n}\right)+o(1)
$$

and

$$
\mathcal{P}\left(u_{n}\right)=\mathcal{P}(\bar{u})+\mathcal{P}\left(v_{n}\right)+o(1) .
$$

Set

$$
\Psi(u)=\frac{1}{N}\|\nabla u\|_{2}^{2}-\frac{1}{2 N} \int_{\mathbb{R}^{N}}(\nabla V(x), x) u^{2} \mathrm{~d} x .
$$

Then it follows from (2.2) with $t=0$ and (2.4) that

$$
\Psi(u) \geq \frac{1-\theta}{N}\|\nabla u\|_{2}^{2}, \quad \forall u \in H^{1}\left(\mathbb{R}^{N}\right) .
$$

Since $I\left(u_{n}\right) \rightarrow m$ and $\mathcal{P}\left(u_{n}\right)=0$, then it follows from (1.2), (1.12), (2.35), (2.36) and (2.37) that

$$
\Psi\left(v_{n}\right)=m-\Psi(\bar{u})+o(1), \quad \mathcal{P}\left(v_{n}\right)=-\mathcal{P}(\bar{u})+o(1)
$$

If there exists a subsequence $\left\{v_{n_{i}}\right\}$ of $\left\{v_{n}\right\}$ such that $v_{n_{i}}=0$, then going to this subsequence, we have

$$
I(\bar{u})=m, \quad \mathcal{P}(\bar{u})=0,
$$

which implies the conclusion of Lemma 2.13 holds. Next, we assume that $v_{n} \neq 0$. We claim that $\mathcal{P}(\bar{u}) \leq 0$. Otherwise $\mathcal{P}(\bar{u})>0$, then (2.39) implies $\mathcal{P}\left(v_{n}\right)<0$ for large $n$. In view of Lemmas 2.6 and 2.7, there exists $t_{n}>0$ such that $\left(v_{n}\right)_{t_{n}} \in \mathcal{M}$. From (1.2), (1.12), (2.3) and (2.39), we obtain

$$
\begin{aligned}
m-\Psi(\bar{u})+o(1) & =\Psi\left(v_{n}\right) \\
& =I\left(v_{n}\right)-\frac{1}{N} \mathcal{P}\left(v_{n}\right) \\
& \geq I\left(\left(v_{n}\right)_{t_{n}}\right)-\frac{t_{n}^{N}}{N} \mathcal{P}\left(v_{n}\right)
\end{aligned}
$$




$$
\geq m-\frac{t_{n}^{N}}{N} \mathcal{P}\left(v_{n}\right) \geq m
$$

which implies $\mathcal{P}(\bar{u}) \leq 0$ due to $\Psi(\bar{u})>0$. Since $\bar{u} \neq 0$ and $\mathcal{P}(\bar{u}) \leq 0$, in view of Lemmas 2.6 and 2.7, there exists $\bar{t}>0$ such that $\bar{u}_{\bar{t}} \in \mathcal{M}$. From (1.2), (1.12), (2.3), (2.37), (2.38) and the weak semicontinuity of norm, one has

$$
\begin{aligned}
m & =\lim _{n \rightarrow \infty}\left[I\left(u_{n}\right)-\frac{1}{N} \mathcal{P}\left(u_{n}\right)\right] \\
& =\lim _{n \rightarrow \infty} \Psi\left(u_{n}\right) \geq \Psi(\bar{u}) \\
& =I(\bar{u})-\frac{1}{N} \mathcal{P}(\bar{u}) \geq I\left(\bar{u}_{\bar{t}}\right)-\frac{\bar{t}^{N}}{N} \mathcal{P}(\bar{u}) \\
& \geq m-\frac{\bar{t}^{N}}{N} \mathcal{P}(\bar{u}) \geq m,
\end{aligned}
$$

which implies (2.40) also holds.

Lemma 2.14. Assume that (V1)-(V3) and (F1)-(F3) hold. If $\bar{u} \in \mathcal{M}($ and $I(\bar{u})=m$, then $\bar{u}$ is a critical point of $I$.

Proof. Assume that $I^{\prime}(\bar{u}) \neq 0$. Then there exist $\delta>0$ and $\varrho>0$ such that

$$
\|u-\bar{u}\| \leq 3 \delta \Rightarrow\left\|I^{\prime}(u)\right\| \geq \varrho .
$$

First, we prove that

$$
\lim _{t \rightarrow 1}\left\|\bar{u}_{t}-\bar{u}\right\|=0 \text {. }
$$

Arguing by contradiction, suppose that there exist $\varepsilon_{0}>0$ and a sequence $\left\{t_{n}\right\}$ such that

$$
\lim _{n \rightarrow \infty} t_{n}=1, \quad\left\|\bar{u}_{t_{n}}-\bar{u}\right\|^{2} \geq \varepsilon_{0} .
$$

Since $\bar{u} \in H^{1}\left(\mathbb{R}^{N}\right)$, there exist $U \in \mathcal{C}_{0}\left(\mathbb{R}^{N}, \mathbb{R}^{N}\right)$ and $v \in \mathcal{C}_{0}\left(\mathbb{R}^{N}, \mathbb{R}\right)$ such that

$$
\int_{\mathbb{R}^{N}}|\nabla \bar{u}-U|^{2}<\frac{\varepsilon_{0}}{20}, \int_{\mathbb{R}^{N}}|\bar{u}-v|^{2}<\frac{\varepsilon_{0}}{20} .
$$

From (2.43) and (2.44), one has

$$
\begin{aligned}
\left\|\nabla \bar{u}_{t_{n}}-\nabla \bar{u}\right\|_{2}^{2} & =\int_{\mathbb{R}^{N}}\left|\nabla \bar{u}_{t_{n}}-\nabla \bar{u}\right|^{2} \mathrm{~d} x \\
& \leq 2 \int_{\mathbb{R}^{N}}\left|\nabla \bar{u}_{t_{n}}-U\right|^{2} \mathrm{~d} x+2 \int_{\mathbb{R}^{N}}|\nabla \bar{u}-U|^{2} \mathrm{~d} x \\
& =2 \int_{\mathbb{R}^{N}}\left|t_{n}^{-1} \nabla \bar{u}\left(t_{n}^{-1} x\right)-U(x)\right|^{2} \mathrm{~d} x+2 \int_{\mathbb{R}^{N}}|\nabla \bar{u}-U|^{2} \mathrm{~d} x \\
& \leq 6 t_{n}^{-2} \int_{\mathbb{R}^{N}}\left|U\left(t_{n}^{-1} x\right)-U(x)\right|^{2} \mathrm{~d} x+6\left|t_{n}^{-1}-1\right|^{2}|U|_{\mathbb{R}^{N}} \mathrm{~d} x+\frac{\left(1+3 t_{n}^{N-2}\right) \varepsilon_{0}}{10} \\
& =\frac{2}{5} \varepsilon_{0}+o(1)
\end{aligned}
$$

and

$$
\begin{aligned}
\left\|\bar{u}_{t_{n}}-\bar{u}\right\|_{2}^{2} & =\int_{\mathbb{R}^{N}}\left|\bar{u}_{t_{n}}-\bar{u}\right|^{2} \mathrm{~d} x \\
& \leq 2 \int_{\mathbb{R}^{N}}\left|\bar{u}_{t_{n}}-v\right|^{2} \mathrm{~d} x+2 \int_{\mathbb{R}^{N}}|\bar{u}-v|^{2} \mathrm{~d} x
\end{aligned}
$$




$$
\begin{aligned}
& \leq 4 \int_{\mathbb{R}^{N}}\left|v\left(t_{n}^{-1} x\right)-v(x)\right|^{2} \mathrm{~d} x+\frac{\left(1+2 t_{n}^{N}\right) \varepsilon_{0}}{10} \\
& =\frac{3}{10} \varepsilon_{0}+o(1) .
\end{aligned}
$$

Combining (2.45) with (2.46), one has

$$
\left\|\bar{u}_{t_{n}}-\bar{u}\right\|^{2}=\left\|\nabla\left(\bar{u}_{t_{n}}\right)-\nabla \bar{u}\right\|_{2}^{2}+\left\|\bar{u}_{t_{n}}-\bar{u}\right\|_{2}^{2} \leq \frac{7}{10} \varepsilon_{0}+o(1) .
$$

(2.47) contradicts with (2.43). Therefore, (2.42) holds. Thus, there exists $\delta_{1} \in(0,1 / 4)$ such that

$$
|t-1|<\delta_{1} \Rightarrow\left\|\bar{u}_{t}-\bar{u}\right\|<\delta
$$

In view of Lemma 2.2, one has

$$
\begin{aligned}
I\left(\bar{u}_{t}\right) & \leq I(\bar{u})-\frac{(1-\theta)\left[2-N t^{N-2}+(N-2) t^{N}\right]}{2 N}\|\nabla \bar{u}\|_{2}^{2} \\
& =m-\frac{(1-\theta) \mathfrak{g}(t)}{2 N}\|\nabla \bar{u}\|_{2}^{2}, \quad \forall t>0 .
\end{aligned}
$$

It follows from (1.12), (2.4) and (2.9) that there exist $T_{1} \in(0,1)$ and $T_{2} \in(1, \infty)$ such that

$$
\mathcal{P}\left(\bar{u}_{T_{1}}\right)>0, \quad \mathcal{P}\left(\bar{u}_{T_{2}}\right)<0 .
$$

Let $\varepsilon:=\min \left\{(1-\theta) \mathfrak{g}\left(T_{1}\right)\|\nabla \bar{u}\|_{2}^{2} / 5 N,(1-\theta) \mathfrak{g}\left(T_{2}\right)\|\nabla \bar{u}\|_{2}^{2} / 5 N, 1, \varrho \delta / 8\right\}$ and $S:=B(\bar{u}, \delta)$. Then [20, Lemma 2.3] yields a deformation $\eta \in \mathcal{C}\left([0,1] \times H^{1}\left(\mathbb{R}^{N}\right), H^{1}\left(\mathbb{R}^{N}\right)\right)$ such that

i) $\quad \eta(1, u)=u$ if $I(u)<m-2 \varepsilon$ or $I(u)>m+2 \varepsilon$;

ii) $\quad \eta\left(1, I^{m+\varepsilon} \cap B(\bar{u}, \delta)\right) \subset I^{m-\varepsilon}$;

iii) $\quad I(\eta(1, u)) \leq I(u), \forall u \in H^{1}\left(\mathbb{R}^{N}\right)$;

iv) $\quad \eta(1, u)$ is a homeomorphism of $H^{1}\left(\mathbb{R}^{N}\right)$.

By Corollary 2.4, $I\left(\bar{u}_{t}\right) \leq I(\bar{u})=m$ for $t>0$, then it follows from (2.48) and ii) that

$$
I\left(\eta\left(1, \bar{u}_{t}\right)\right) \leq m-\varepsilon, \quad \forall t>0,|t-1|<\delta_{1} .
$$

On the other hand, by iii) and (2.49), one has

$$
\begin{aligned}
I\left(\eta\left(1, \bar{u}_{t}\right)\right) & \leq I\left(\bar{u}_{t}\right) \\
& \leq m-\frac{(1-\theta) \mathfrak{g}(t)}{2 N}\|\nabla \bar{u}\|_{2}^{2} \\
& \leq m-\frac{(1-\theta) \delta_{2}}{2 N}\|\nabla \bar{u}\|_{2}^{2}, \quad \forall t>0, \quad|t-1| \geq \delta_{1},
\end{aligned}
$$

where

$$
\delta_{2}:=\min \left\{\mathfrak{g}\left(1-\delta_{1}\right), \mathfrak{g}\left(1+\delta_{1}\right)\right\}>0 .
$$

Combining (2.51) with (2.52), we have

$$
\max _{t \in\left[T_{1}, T_{2}\right]} I\left(\eta\left(1, \bar{u}_{t}\right)\right)<m .
$$

Define $\Psi_{0}(t):=\mathcal{P}\left(\eta\left(1, \bar{u}_{t}\right)\right)$ for $t>0$. It follows from (2.49) and i) that $\eta\left(1, \bar{u}_{t}\right)=\bar{u}_{t}$ for $t=T_{1}$ and $t=T_{2}$, which, together with (2.50), implies

$$
\Psi_{0}\left(T_{1}\right)=\mathcal{P}\left(\bar{u}_{T_{1}}\right)>0, \quad \Psi_{0}\left(T_{2}\right)=\mathcal{P}\left(\bar{u}_{T_{1}}\right)<0 .
$$

Since $\Psi_{0}(t)$ is continuous on $(0, \infty)$, then we have that $\eta\left(1, \bar{u}_{t}\right) \cap \mathcal{M} \neq \emptyset$ for some $t_{0} \in\left[T_{1}, T_{2}\right]$, contradicting to the definition of $m$.

Proof of Theorem 1.2. In view of Lemmas 2.9, 2.13 and 2.14, there exists $\bar{u} \in \mathcal{M}$ such that

$$
I(\bar{u})=m=\inf _{u \in \Lambda} \max _{t>0} I\left(u_{t}\right), \quad I^{\prime}(\bar{u})=0 .
$$

This shows that $\bar{u}$ is a ground state solution of Pohožaev type for (1.1). 


\section{The least energy solutions for (1.1)}

In this section, we give the proof of Theorem 1.5.

Proposition 3.1. [8] Let $X$ be a Banach space and let $J \subset \mathbb{R}^{+}$be an interval, and

$$
\Phi_{\lambda}(u)=A(u)-\lambda B(u), \quad \forall \lambda \in J,
$$

be a family of $\mathrm{C}^{1}$-functional on $X$ such that

i) either $A(u) \rightarrow+\infty$ or $B(u) \rightarrow+\infty$, as $\|u\| \rightarrow \infty$;

ii) $\quad B$ maps every bounded set of $X$ into a set of $\mathbb{R}$ bounded below;

iii) there are two points $v_{1}, v_{2}$ in $X$ such that

$$
\tilde{c}_{\lambda}:=\inf _{y \in \tilde{\Gamma}} \max _{t \in[0,1]} \Phi_{\lambda}(y(t))>\max \left\{\Phi_{\lambda}\left(v_{1}\right), \Phi_{\lambda}\left(v_{2}\right)\right\},
$$

where

$$
\tilde{\Gamma}=\left\{y \in \mathcal{C}([0,1], X): y(0)=v_{1}, y(1)=v_{2}\right\} .
$$

Then, for almost every $\lambda \in J$, there exists a sequence $\left\{u_{n}(\lambda)\right\}$ such that

i) $\quad\left\{u_{n}(\lambda)\right\}$ is bounded in $X$;

ii) $\quad \Phi_{\lambda}\left(u_{n}(\lambda)\right) \rightarrow c_{\lambda}$;

iii) $\quad \Phi_{\lambda}^{\prime}\left(u_{n}(\lambda)\right) \rightarrow 0$ in $X^{\star}$, where $X^{\star}$ is the dual of $X$.

Lemma 3.2. [7] Assume that (V1), (V2), (F1) and (F2) hold. Let $u$ be a critical point of $I_{\lambda}$ in $H^{1}\left(\mathbb{R}^{N}\right)$, then we have the following Pohožaev type identity

$$
\begin{aligned}
\mathcal{P}_{\lambda}(u):= & \frac{N-2}{2}\|\nabla u\|_{2}^{2}+\frac{1}{2} \int_{\mathbb{R}^{N}}[N V(x)+\nabla V(x) \cdot x] u^{2} \mathrm{~d} x \\
& -N \lambda \int_{\mathbb{R}^{N}} F(u) \mathrm{d} x=0 .
\end{aligned}
$$

By Corollary 2.3, we have the following lemma.

Lemma 3.3. Assume that (F1) and (F2) hold. Then

$$
\begin{gathered}
I_{\lambda}^{\infty}(u)=I_{\lambda}^{\infty}\left(u_{t}\right)+\frac{1-t^{N}}{N} \mathcal{P}_{\lambda}^{\infty}(u)+\frac{2-N t^{N-2}+(N-2) t^{N}}{2 N}\|\nabla u\|_{2}^{2}, \\
\forall u \in H^{1}\left(\mathbb{R}^{N}\right), \quad t>0, \quad \lambda \geq 0 .
\end{gathered}
$$

In view of Theorem 1.3, $I_{1}^{\infty}=I^{\infty}$ has a minimizer $u_{1}^{\infty} \neq 0$ on $\mathcal{M}_{1}^{\infty}=\mathcal{M}^{\infty}$, i.e.

$$
u_{1}^{\infty} \in \mathcal{M}_{1}^{\infty}, \quad\left(I_{1}^{\infty}\right)^{\prime}\left(u_{1}^{\infty}\right)=0 \quad \text { and } \quad m_{1}^{\infty}=I_{1}^{\infty}\left(u_{1}^{\infty}\right),
$$

where $m_{\lambda}^{\infty}$ is defined by (1.18). Since (1.3) is autonomous, $V \in \mathcal{C}\left(\mathbb{R}^{N}, \mathbb{R}\right)$ and $V(x) \leq V_{\infty}$ but $V(x) \equiv V_{\infty}$, then there exist $\bar{x} \in \mathbb{R}^{N}$ and $\bar{r}>0$ such that

$$
V_{\infty}-V(x)>0,\left|u_{1}^{\infty}(x)\right|>0 \text { a.e. }|x-\bar{x}| \leq \bar{r} .
$$

Lemma 3.4. Assume that (V1), (V2) and (F1)-(F3) hold. Then 
(i) there exists $T>0$ independent of $\lambda$ such that $I_{\lambda}\left(\left(u_{1}^{\infty}\right)_{T}\right)<0$ for all $\lambda \in[0.5,1]$;

(ii) there exists a positive constant $\kappa_{0}$ independent of $\lambda$ such that for all $\lambda \in[0.5,1]$,

$$
c_{\lambda}:=\inf _{y \in \Gamma} \max _{t \in[0,1]} I_{\lambda}(y(t)) \geq \kappa_{0}>\max \left\{I_{\lambda}(0), I_{\lambda}\left(\left(u_{1}^{\infty}\right)_{T}\right)\right\}
$$

where

$$
\Gamma=\left\{y \in \mathcal{C}\left([0,1], H^{1}\left(\mathbb{R}^{N}\right)\right): y(0)=0, y(1)=\left(u_{1}^{\infty}\right)_{T}\right\} ;
$$

(iii) $\quad c_{\lambda}$ is bounded for $\lambda \in[0.5,1]$;

(iv) $m_{\lambda}^{\infty}$ is non-increasing on $\lambda \in[0.5,1]$;

(v) $\quad \lim \sup _{\lambda \rightarrow \lambda_{0}} c_{\lambda} \leq c_{\lambda_{0}}$ for $\lambda_{0} \in(0.5,1]$.

Since $m_{\lambda}^{\infty}=I_{\lambda}^{\infty}\left(u_{\lambda}^{\infty}\right)$ and $\int_{\mathbb{R}^{N}} F\left(u_{\lambda}^{\infty}\right) \mathrm{d} x>0$, then the proofs of (i)-(iv) in Lemma 3.4 is standard, (v) can be proved similar to [5, Lemma 2.3], so we omit it.

Lemma 3.5. Assume that (V1), (V2) and (F1)-(F3) hold. Then there exists $\bar{\lambda} \in[1 / 2,1)$ such that $c_{\lambda}<m_{\lambda}^{\infty}$ for $\lambda \in(\bar{\lambda}, 1]$.

Proof. It is easy to see that $I_{\lambda}\left(\left(u_{1}^{\infty}\right)_{t}\right)$ is continuous on $t \in(0, \infty)$. Hence for any $\lambda \in[1 / 2,1]$, we can choose $t_{\lambda} \in(0, T)$ such that $I_{\lambda}\left(\left(u_{1}^{\infty}\right)_{t_{\lambda}}\right)=\max _{t \in[0, T]} I_{\lambda}\left(\left(u_{1}^{\infty}\right)_{t}\right)$. Setting

$$
y_{0}(t)= \begin{cases}\left(u_{1}^{\infty}\right)_{(t T)}, & \text { for } t>0 \\ 0, & \text { for } t=0\end{cases}
$$

Then $y_{0} \in \Gamma$ defined by Lemma 3.4 (ii). Moreover

$$
I_{\lambda}\left(\left(u_{1}^{\infty}\right)_{t_{\lambda}}\right)=\max _{t \in[0,1]} I_{\lambda}\left(y_{0}(t)\right) \geq c_{\lambda}
$$

Let

$$
\zeta_{0}:=\min \{3 \bar{r} / 8(1+|\bar{x}|), 1 / 4\} .
$$

Then it follows from (3.5) and (3.7) that

$$
|x-\bar{x}| \leq \frac{\bar{r}}{2} \text { and } s \in\left[1-\zeta_{0}, 1+\zeta_{0}\right] \Rightarrow|s x-\bar{x}| \leq \bar{r} .
$$

Since $\mathcal{P}^{\infty}\left(u_{1}^{\infty}\right)=0$, then $\int_{\mathbb{R}^{N}} F\left(u_{1}^{\infty}\right) \mathrm{d} x>0$. Let

$$
\begin{aligned}
\bar{\lambda}:= & \max \left\{\frac{1}{2}, 1-\frac{\left(1-\zeta_{0}\right)^{N} \min _{s \in\left[1-\zeta_{0}, 1+\zeta_{0}\right]} \int_{\mathbb{R}^{N}}\left[V_{\infty}-V(s x)\right]\left|u_{1}^{\infty}\right|^{2} \mathrm{~d} x}{T^{N} \int_{\mathbb{R}^{N}} F\left(u_{1}^{\infty}\right) \mathrm{d} x},\right. \\
& \left.1-\frac{\min \left\{\mathfrak{g}\left(1-\zeta_{0}\right), \mathfrak{g}\left(1+\zeta_{0}\right)\right\}\left\|\nabla u_{1}^{\infty}\right\|_{2}^{2}}{N T^{N} \int_{\mathbb{R}^{N}} F\left(u_{1}^{\infty}\right) \mathrm{d} x}\right\} .
\end{aligned}
$$

Then it follows from (2.1), (3.5) and (3.8) that $1 / 2 \leq \bar{\lambda}<1$. We have two cases to distinguish:

Case i). $t_{\lambda} \in\left[1-\zeta_{0}, 1+\zeta_{0}\right]$. From (1.14), (1.15), (3.3)-(3.6), (3.8), (3.9) and Lemma 3.4 (iv), we have

$$
\begin{aligned}
m_{\lambda}^{\infty} & \geq m_{1}^{\infty}=I_{1}^{\infty}\left(u_{1}^{\infty}\right) \geq I_{1}^{\infty}\left(\left(u_{1}^{\infty}\right)_{t_{\lambda}}\right) \\
& =I_{\lambda}\left(\left(u_{1}^{\infty}\right)_{t_{\lambda}}\right)-\frac{(1-\lambda) t_{\lambda}^{N}}{2} \int_{\mathbb{R}^{N}} F\left(u_{1}^{\infty}\right) \mathrm{d} x+\frac{t_{\lambda}^{N}}{2} \int_{\mathbb{R}^{N}}\left[V_{\infty}-V\left(t_{\lambda} x\right)\right]\left|u_{1}^{\infty}\right|^{2} \mathrm{~d} x \\
& \geq c_{\lambda}-\frac{(1-\lambda) T_{0}^{N}}{2} \int_{\mathbb{R}^{N}} F\left(u_{1}^{\infty}\right) \mathrm{d} x
\end{aligned}
$$




$$
\begin{aligned}
& +\frac{\left(1-\zeta_{0}\right)^{N}}{2} \min _{s \in\left[1-\zeta_{0}, 1+\zeta_{0}\right]} \int_{\mathbb{R}^{N}}\left[V_{\infty}-V(s x)\right]\left|u_{1}^{\infty}\right|^{2} \mathrm{~d} x \\
>\quad & c_{\lambda}, \quad \forall \lambda \in(\bar{\lambda}, 1] .
\end{aligned}
$$

Case ii). $t_{\lambda} \in\left(0,1-\zeta_{0}\right) \cup\left(1+\zeta_{0}, T\right)$. From (V2), (1.14), (1.15), (2.1), (3.3), (3.4), (3.6), (3.9) and Lemma 3.4 (iv), we have

$$
\begin{aligned}
m_{\lambda}^{\infty} & \geq m_{1}^{\infty}=I_{1}^{\infty}\left(u_{1}^{\infty}\right) \geq I_{1}^{\infty}\left(\left(u_{1}^{\infty}\right)_{t_{\lambda}}\right)+\frac{\mathfrak{g}\left(t_{\lambda}\right)\left\|\nabla u_{1}^{\infty}\right\|_{2}^{2}}{2 N} \\
& =I_{\lambda}\left(\left(u_{1}^{\infty}\right)_{t_{\lambda}}\right)-\frac{(1-\lambda) t_{\lambda}^{N}}{2} \int_{\mathbb{R}^{N}} F\left(u_{1}^{\infty}\right) \mathrm{d} x+\frac{t_{\lambda}^{N}}{2} \int_{\mathbb{R}^{N}}\left[V_{\infty}-V\left(t_{\lambda} x\right)\right]\left|u_{1}^{\infty}\right|^{2} \mathrm{~d} x+\frac{\mathfrak{g}\left(t_{\lambda}\right)\left\|\nabla u_{1}^{\infty}\right\|_{2}^{2}}{2 N} \\
& \geq c_{\lambda}-\frac{(1-\lambda) T^{N}}{2} \int_{\mathbb{R}^{N}} F\left(u_{1}^{\infty}\right) \mathrm{d} x+\frac{\min \left\{\mathfrak{g}\left(1-\zeta_{0}\right), \mathfrak{g}\left(1+\zeta_{0}\right)\right\}\left\|\nabla u_{1}^{\infty}\right\|_{2}^{2}}{2 N} \\
& >c_{\lambda}, \quad \forall \lambda \in(\bar{\lambda}, 1] .
\end{aligned}
$$

In both cases, we obtain that $c_{\lambda}<m_{\lambda}^{\infty}$ for $\lambda \in(\bar{\lambda}, 1]$.

Lemma 3.6. [7] Assume that (V1), (V2) and (F1)-(F3) hold. Let $\left\{u_{n}\right\}$ be a bounded (PS) sequence for $I_{\lambda}$, for $\lambda \in[1 / 2,1]$. Then there exists a subsequence of $\left\{u_{n}\right\}$, still denoted by $\left\{u_{n}\right\}$, an integer $l \in \mathbb{N} \cup\{0\}$, a sequence $\left\{y_{n}^{k}\right\}$ and $w^{k} \in H^{1}\left(\mathbb{R}^{3}\right)$ for $1 \leq k \leq l$, such that

(i) $\quad u_{n} \rightarrow u_{0}$ with $I_{\lambda}^{\prime}\left(u_{0}\right)=0$;

(ii) $\quad w^{k} \neq 0$ and $\left(I_{\lambda}^{\infty}\right)^{\prime}\left(w^{k}\right)=0$ for $1 \leq k \leq l$;

(iii) $\quad\left\|u_{n}-u_{0}-\sum_{k=1}^{l} w^{k}\left(\cdot+y_{n}^{k}\right)\right\| \rightarrow 0$;

(iv) $\quad I_{\lambda}\left(u_{n}\right) \rightarrow I_{\lambda}\left(u_{0}\right)+\sum_{i=1}^{l} I_{\lambda}^{\infty}\left(w^{i}\right)$;

where we agree that in the case $l=0$ the above holds without $w^{k}$.

Lemma 3.7. Assume that (V1), (V2), (V4) and (F1)-(F3) hold.Then for almost every $\lambda \in(\bar{\lambda}, 1]$, there exists $u_{\lambda} \in H^{1}\left(\mathbb{R}^{N}\right) \backslash\{0\}$ such that

$$
I_{\lambda}^{\prime}\left(u_{\lambda}\right)=0, \quad I_{\lambda}\left(u_{\lambda}\right)=c_{\lambda} .
$$

Proof. Under (V1), (V2) and (F1)-(F3), Lemma 3.4 implies that $I_{\lambda}(u)$ satisfies the assumptions of Proposition 3.1 with $X=H^{1}\left(\mathbb{R}^{N}\right), J=[\bar{\lambda}, 1]$ and $\Phi_{\lambda}=I_{\lambda}$. So for almost every $\lambda \in(\bar{\lambda}, 1]$, there exists a bounded sequence $\left\{u_{n}(\lambda)\right\} \subset H^{1}\left(\mathbb{R}^{N}\right)$ (for simplicity, we denote the sequence by $\left\{u_{n}\right\}$ instead of $\left\{u_{n}(\lambda)\right\}$ ) such that

$$
I_{\lambda}\left(u_{n}\right) \rightarrow c_{\lambda}>0, \quad I_{\lambda}^{\prime}\left(u_{n}\right) \rightarrow 0 .
$$

By Lemmas 3.2 and 3.6, there exist a subsequence of $\left\{u_{n}\right\}$, still denoted by $\left\{u_{n}\right\}$, and $u_{\lambda} \in H^{1}\left(\mathbb{R}^{N}\right)$, an integer $l \in \mathbb{N} \cup\{0\}$, and $w^{1}, \ldots, w^{l} \in H^{1}\left(\mathbb{R}^{N}\right) \backslash\{0\}$ such that

$$
\begin{gathered}
u_{n} \rightarrow u_{\lambda} \text { in } H^{1}\left(\mathbb{R}^{N}\right), \quad I_{\lambda}^{\prime}\left(u_{\lambda}\right)=0, \\
\left(I_{\lambda}^{\infty}\right)^{\prime}\left(w^{k}\right)=0, \quad I_{\lambda}^{\infty}\left(w^{k}\right) \geq m_{\lambda}^{\infty}, \quad 1 \leq k \leq l
\end{gathered}
$$

and

$$
c_{\lambda}=I_{\lambda}\left(u_{\lambda}\right)+\sum_{k=1}^{l} I_{\lambda}^{\infty}\left(w^{k}\right) .
$$

Since $I_{\lambda}^{\prime}\left(u_{\lambda}\right)=0$, then it follows from Lemma 3.2 that

$$
\mathcal{P}_{\lambda}\left(u_{\lambda}\right)=\frac{N-2}{2}\left\|\nabla u_{\lambda}\right\|_{2}^{2}+\frac{1}{2} \int_{\mathbb{R}^{N}}[N V(x)+\nabla V(x) \cdot x] u_{\lambda}^{2} \mathrm{~d} x
$$




$$
-N \lambda \int_{\mathbb{R}^{N}} F\left(u_{\lambda}\right) \mathrm{d} x=0
$$

Since $\left\|u_{n}\right\| \nrightarrow 0$, we deduce from (3.13) and (3.14) that if $u_{\lambda}=0$ then $l \geq 1$ and

$$
c_{\lambda}=I_{\lambda}\left(u_{\lambda}\right)+\sum_{k=1}^{l} I_{\lambda}^{\infty}\left(w^{k}\right) \geq m_{\lambda}^{\infty},
$$

which contradicts with Lemma 3.5. Thus $u_{\lambda} \neq 0$. It follows from (1.14), (2.4), (3.15) and (V4) that

$$
\begin{aligned}
I_{\lambda}\left(u_{\lambda}\right) & =I_{\lambda}\left(u_{\lambda}\right)-\frac{1}{N} \mathcal{P}_{\lambda}\left(u_{\lambda}\right) \\
& =\frac{1}{N}\left\|\nabla u_{\lambda}\right\|_{2}^{2}-\frac{1}{2 N} \int_{\mathbb{R}^{N}}(\nabla V(x), x) u_{\lambda}^{2} \mathrm{~d} x \geq \frac{1-\theta}{N}\left\|\nabla u_{\lambda}\right\|_{2}^{2}>0 .
\end{aligned}
$$

From (3.14) and (3.16), one has

$$
c_{\lambda}=I_{\lambda}\left(u_{\lambda}\right)+\sum_{k=1}^{l} I_{\lambda}^{\infty}\left(w^{k}\right)>\operatorname{lm}_{\lambda}^{\infty} .
$$

By Lemma 3.5, we have $c_{\lambda}<m_{\lambda}^{\infty}$ for $\lambda \in(\bar{\lambda}, 1]$, which, together with (3.17), implies that $l=0$ and $I_{\lambda}\left(u_{\lambda}\right)=$ $c_{\lambda}$.

Lemma 3.8. Assume that (V1), (V2), (V4) and (F1)-(F3) hold. Then there exists $\bar{u} \in H^{1}\left(\mathbb{R}^{N}\right) \backslash\{0\}$ such that

$$
I^{\prime}(\bar{u})=0, \quad 0<I(\bar{u})<c_{1} .
$$

Proof. In view of Lemma 3.7, there exist two sequences $\left\{\lambda_{n}\right\} \subset[\bar{\lambda}, 1]$ and $\left\{u_{\lambda_{n}}\right\} \subset H^{1}\left(\mathbb{R}^{N}\right) \backslash\{0\}$, denoted by $\left\{u_{n}\right\}$, such that

$$
\lambda_{n} \rightarrow 1, \quad c_{\lambda_{n}} \rightarrow c_{\star}, \quad I_{\lambda_{n}}^{\prime}\left(u_{n}\right)=0, \quad 0<I_{\lambda_{n}}\left(u_{n}\right) \leq c_{\lambda_{n}} .
$$

Then it follows from Lemma 3.2 that

$$
\begin{gathered}
\mathcal{P}_{\lambda_{n}}\left(u_{n}\right):=\frac{N-2}{2}\left\|\nabla u_{n}\right\|_{2}^{2}+\frac{1}{2} \int_{\mathbb{R}^{N}}[N V(x)+\nabla V(x) \cdot x] u_{n}^{2} \mathrm{~d} x \\
-N \lambda_{n} \int_{\mathbb{R}^{N}} F\left(u_{n}\right) \mathrm{d} x=0 .
\end{gathered}
$$

From (V4), (1.14), (2.4), (3.19), (3.20) and Lemma 3.4 (iii), one has

$$
\begin{aligned}
C_{5} & \geq c_{\lambda_{n}}=I_{\lambda_{n}}\left(u_{n}\right)-\frac{1}{N} \mathcal{P}_{\lambda_{n}}\left(u_{n}\right) \\
& =\frac{1}{N}\left\|\nabla u_{n}\right\|_{2}^{2}-\frac{1}{2 N} \int_{\mathbb{R}^{N}} \nabla V(x) \cdot x u_{n}^{2} \mathrm{~d} x \\
& \geq \frac{1-\theta}{N}\left\|\nabla u_{n}\right\|_{2}^{2} .
\end{aligned}
$$

This shows that $\left\{\left\|\nabla u_{n}\right\|_{2}\right\}$ is bounded. Next, we demonstrate that $\left\{u_{n}\right\}$ is bounded in $H^{1}\left(\mathbb{R}^{N}\right)$. According to (V1) and (V2), it is easy to show that there exists a constant $y_{3}>0$ such that

$$
\int_{\mathbb{R}^{N}}\left[|\nabla u|^{2}+V(x) u^{2}\right] \mathrm{d} x \geq y_{3}\|u\|^{2}, \quad \forall u \in H^{1}\left(\mathbb{R}^{N}\right) .
$$

From (F1), (F2), (1.14), (3.19), (3.21), (3.22), Lemma 3.4 (iii) and the Sobolev embedding theorem, we have

$$
y_{3}\left\|u_{n}\right\|^{2} \leq \int_{\mathbb{R}^{N}}\left[\left|\nabla u_{n}\right|^{2}+V(x) u_{n}^{2}\right] \mathrm{d} x
$$




$$
\begin{aligned}
& =2 c_{\lambda_{n}}+2 \lambda_{n} \int_{\mathbb{R}^{N}} F\left(u_{n}\right) \mathrm{d} x \\
& \leq 2 C_{5}+\frac{y_{3}}{2}\left\|u_{n}\right\|^{2}+C_{6}\left\|u_{n}\right\|_{2^{*}}^{2^{*}} \\
& \leq 2 C_{5}+\frac{y_{3}}{2}\left\|u_{n}\right\|^{2}+C_{6} S^{-2^{*} / 2}\left\|\nabla u_{n}\right\|_{2}^{2^{*}} .
\end{aligned}
$$

Hence, $\left\{u_{n}\right\}$ is bounded in $H^{1}\left(\mathbb{R}^{N}\right)$. In view of Lemma $3.4(\mathrm{v})$, we have $\lim _{n \rightarrow \infty} c_{\lambda_{n}}=c_{\star} \leq c_{1}$. Hence, it follows from (1.2), (1.14) and (3.19) that

$$
I\left(u_{n}\right) \rightarrow c_{\star}, \quad I^{\prime}\left(u_{n}\right) \rightarrow 0 .
$$

This shows that $\left\{u_{n}\right\}$ satisfy (3.11) with $c_{\lambda}=c_{\star}$. In view of the proof of Lemma 3.7, we can show that there exists $\bar{u} \in H^{1}\left(\mathbb{R}^{N}\right) \backslash\{0\}$ such that (3.18) holds.

Proof of Theorem 1.5. Let

$$
\mathcal{K}:=\left\{u \in H^{1}\left(\mathbb{R}^{N}\right) \backslash\{0\}: I^{\prime}(u)=0\right\}, \quad \hat{m}:=\inf _{u \in \mathcal{K}} I(u) .
$$

Then Lemma 3.8 shows that $\mathcal{K} \neq \emptyset$ and $\hat{m} \leq c_{1}$. For any $u \in \mathcal{K}$, Lemma 3.2 implies $\mathcal{P}(u)=\mathcal{P}_{1}(u)=0$. Hence it follows from (3.16) that $I(u)=I_{1}(u)>0$, and so $\hat{m} \geq 0$. Let $\left\{u_{n}\right\} \subset \mathcal{K}$ such that

$$
I^{\prime}\left(u_{n}\right)=0, \quad I\left(u_{n}\right) \rightarrow \hat{m} .
$$

In view of Lemma 3.5, $\hat{m} \leq c_{1}<m_{1}^{\infty}$. By a similar argument as in the proof of Lemma 3.7, we can prove that there exists $\bar{u} \in H^{1}\left(\mathbb{R}^{N}\right) \backslash\{0\}$ such that

$$
I^{\prime}(\bar{u})=0, \quad I(\bar{u})=\hat{m} .
$$

This shows that $\bar{u}$ is a nontrivial least energy solution of (1.1).

Acknowledgement: This work was partially supported by the National Natural Science Foundation of China (11571370).

\section{References}

[1] H. Berestycki, P. L. Lions, Nonlinear scalar field equations, I. Existence of a ground state, Arch. Rational Mech. Anal. 82, (1983), 313-345.

[2] S. T. Chen and X. H. Tang, Improved results for Klein-Gordon-Maxwell systems with general nonlinearity, Disc. Contin. Dyn. Syst. A 38, (2018), 2333-2348.

[3] S. T. Chen and X. H. Tang, Ground state solutions for generalized quasilinear Schrödinger equations with variable potentials and Berestycki-Lions nonlinearities, J. Math. Phys. 59, (2018), no. 081508, 1-18

[4] S. T. Chen, B. L. Zhang, X. H. Tang, Existence and non-existence results for Kirchhoff-type problems with convolution nonlinearity, Adv. Nonlinear Anal. (2018), https://doi.org/10.1515/anona-2018-0147

[5] L. Jeanjean, On the existence of bounded Palais-Smale sequences and application to a Landesman- Lazer-type problem set on $\mathbb{R}^{N}$, Proc. Roy. Soc. Edinburgh Sect. A 129, (1999), 787-809.

[6] L. Jeanjean, K. Tanka, A remark on least energy solutions in $\mathbb{R}^{N}$, Proc. Amer. Math. Soc. 131, (2003), 2399-2408.

[7] L. Jeanjean, K. Tanka, A positive solution for a nonlinear Schrödinger equation on $\mathbb{R}^{N}$, Indiana Univ. Math. J. 54, (2005), 443-464.

[8] L. Jeanjean and J. F. Toland, Bounded Palais-Smale mountain-pass sequences, C. R. Acad. Sci. Paris Sér. I Math. 327, (1998), 23-28.

[9] S. I. Pohožaev, Eigenfunctions of the equation $a u+2 f(u)=0$, Sov. Math. Doklady 5, (1965), 1408-1411.

[10] P. H. Rabinowitz, On a class of nonlinear Schrödinger equations, Z. Angew. Math. Phys. 43, (1992), 270-291.

[11] Y. H. Sato, M. Shibata, Existence of a positive solution for nonlinear Schrödinger equations with general nonlinearity, $A d v$. Nonlinear Anal. 3, (2014), 55-67.

[12] J. Shatah, Unstable ground state of nonlinear Klein-Gordon equations, Trans. Amer. Math. Soc. 290, (1985), 701-710.

[13] M. Struwe, Variational methods, Results in Mathematics and Related Areas, 3, Springer-Verlag, Berlin, 1996. 
[14] X. H. Tang and S. T. Chen, Ground state solutions of Nehari-Pohožaev type for Kirchhoff-type problems with general potentials, Calc. Var. Partial Differential Equations 56, (2017), no. 4, 1-25.

[15] X. H. Tang and S. T. Chen, Ground state solutions of Nehari-Pohožaev type for Schrödinger-Poisson problems with general potentials, Disc. Contin. Dyn. Syst. A 37, (2017), 4973-5002.

[16] X. H. Tang and X. Y. Lin, Existence of ground state solutions of Nehari-Pankov type to Schrödinger systems, Sci. China Math 62, (2019), https://doi.org/10.1007/s11425-017-9332-3.

[17] X. H. Tang, X. Lin, J. Yu, Nontrivial solutions for Schrödinger equation with local super-quadratic conditions, J. Dyn. Differ. Equ, (2018), 1-15. https://doi.org/10.1007/s10884-018-9662-2.

[18] X. H. Tang, S. T. Chen, Singularly perturbed choquard equations with nonlinearity satisfying Berestycki-Lions assumptions, Adv. Nonlinear Anal., https://doi.org/10.1515/anona-2020-0007

[19] A. Vincenzo, Zero mass case for a fractional Berestycki-Lions-type problem, Adv. Nonlinear Anal. 7, (2018), 365-374.

[20] M. Willem, Minimax theorems, Progress in Nonlinear Differential Equations and their Applications, 24, Birkhäuser Boston Inc., Boston, MA, 1996. 